\title{
Barbey d'Aurevilly et la modernité, textes réunis par Philippe Berthier
}

\section{Ida Merello}

\section{(2) OpenEdition}

1 Journals

\section{Edizione digitale}

URL: http://journals.openedition.org/studifrancesi/5712

DOI: ERREUR PDO dans /localdata/www-bin/Core/Core/Db/Db.class.php L.34 : SQLSTATE[HY000]

[2006] MySQL server has gone away

ISSN: 2421-5856

\section{Editore}

Rosenberg \& Sellier

\section{Edizione cartacea}

Data di pubblicazione: 1 septembre 2011

Paginazione: $442-443$

ISSN: 0039-2944

\section{Notizia bibliografica digitale}

Ida Merello, «Barbey d'Aurevilly et la modernité, textes réunis par Philippe Berthier», Studi Francesi [Online], 164 (LV | II) | 2011, online dal 30 novembre 2015, consultato il 07 janvier 2021. URL: http:// journals.openedition.org/studifrancesi/5712 ; DOI: https://doi.org/ERREUR PDO dans /localdata/ www-bin/Core/Core/Db/Db.class.php L.34 : SQLSTATE[HY000] [2006] MySQL server has gone away

Questo documento è stato generato automaticamente il 7 janvier 2021.

\section{(c) $($ ) $\odot \odot$}

Studi Francesi è distribuita con Licenza Creative Commons Attribuzione - Non commerciale - Non opere derivate 4.0 Internazionale. 


\title{
Barbey d'Aurevilly et la modernité, textes réunis par Philippe Berthier
}

\author{
Ida Merello
}

\section{NOTIZIA}

AA. VV., Barbey d'Aurevilly et la modernité, Colloque du Bicentenaire (1808-2008), textes réunis par Philippe BERTHIER, Paris, Champion, 344 pp.

1 Laurence CLAUDE-PHALIPPOU (Quand le désir (dé)fait la modernité, pp. 9-20) mette sul tappeto la questione centrale, ossia quale sia il concetto di modernità per Barbey, e lo riassume nel sentimento di uno scacco in tutte le sue forme: continua delusione di aspettative, incapacità di generare la narrazione. Per questo la finzione può trattarne poco, a favore invece di un discorso capace di innescare il desiderio, «filomitico».

2 Hugues LAROCHE (La modernité et sa figure, pp. 21-30) nota che le sessantasei occorrenze di moderno nell'opera di Barbey sono legate a tutto quello che lui odia (realismo, filantropismo, naturalismo, umanesimo, positivismo, democrazia, socialismo) in quanto contrario alla sua utopia di una società teocratica. Barbey apprezza i moderni (Vigny, Balzac, Baudelaire) nel momento in cui sono critici del proprio tempo. Per questo, conclude l'A., Barbey sceglie nei romanzi il tempo appena trascorso, che precede la rovina; mentre la modernità si installa nel post-rovina.

3 Méké MÉITÉ (Barbey d'Aurevilly et le roman historique: quelle modernité?, pp. 31-44) riprende la definizione di modernità di Benjamin (ciò che si iscrive in un'epoca) e la incarna in Barbey nella scelta dell'istanza narrativa di rappresentare la storia attraverso la sua poeticizzazione.

4 Josette soutet (Sous le regard de Dionysos, pp. 45-60) legge in chiave mitocriptica Le chevalier Des Touches, vedendovi la riscrittura del mito di Dioniso, per cui la Rivoluzione francese è la ripetizione di una scena primitiva.

5 Céline BRICAULT (Barbey d'Aurevilly, «Janus, gardien du seuil»: modernité d' "Un prêtre marié», pp.61-76) interpreta la concezione di modernità di Barbey come una tensione tra il 
desiderio di libertà e il bisogno di legami. La modernità storica è quasi sempre vista come rivoluzionaria e catastrofica, come rappresentazione di un mondo in declino, perciò la tendenza comune dei personaggi è quella di tenersi a distanza da un contesto politico di cui pure sono i rappresentanti.

6 Alice DE GEORGES-MÉTRAL (Holisme et rhizome dans «Un prêtre marié»: modernité d'un antimoderne, pp. 77-92) fa coincidere l'atteggiamento anti moderno di Barbey con una postura da dandy e un impegno cattolico legittimista: nel dialogo tra due visioni olistiche del mondo, quella dei diritti umani e quella dei diritti divini e monarchici, la prima rinnega i valori del passato e la seconda si illude che siano ancora possibili, arrivando a scontri che Barbey traduce nella metafora del filo spezzato. L'A. legge il romanzo in questione come la linea di fuga espressione di due serie segmentate.

7 L'intervento di Didier РніLіргот (Les diaboliques, où les histoires tragiques de notre temps, pp.93-174) appare per l'ampiezza piuttosto un intero volume inserito in una miscellanea. L'A. stabilisce un'analogia tra Les Diaboliques e il genere delle Histoires tragiques del XVII secolo, non solo sulla base della definizione stessa di Barbey relativa alla propria opera, ma anche dopo un'accurata disamina delle costanti del genere, individuate nella brevità, nel principio della raccolta, nella tematica dell'eros tragico, opposto all'amore per la sua complessità passionale, nella rapida mutazione dei sentimenti dall'amore all'odio. Inoltre, tragicità diventa sinonimo di verità, e di verità mostruosa e incredibile, in opposizione alla verosimiglianza: come nelle Histoires tragiques, Barbey mette in scena un teatro della crudeltà a scopo edificante come esigenza di attualità in un contesto storico di disordine e di crisi. L'A. risolve la questione se Barbey abbia o meno conosciuto direttamente i modelli secenteschi, indicandone comunque la mediazione certa attraverso le Chroniques italiennes di Stendhal, che rappresenta un testo di riferimento insieme ai Contes bruns di Balzac. In entrambi Barbey può ritrovare la poetica del racconto/conversazione, dell'aneddoto vero o presentato come tale, del racconto/testimonianza nell'ambito di una poetica e di una materia propriamente tragica. L'A. cita inoltre come elemento fondamentale per il ritorno alla sorgente dell'histoire tragique il fatto che il romanticismo è per sua natura shakespeariano, e, se Barbey adora Shakespeare, è noto che l'autore inglese si era interessato all'histoire tragique francese. Per l'A. comunque Barbey ritrova l'invenzione del racconto tragico per una necessità profonda del suo immaginario e un'esigenza altrettanto profonda della sua poetica, che investe tutta la sua opera. L'A. considera a questo proposito esemplare il preambolo di La Vengeance d'une femme, che non solo ha valore di postfazione delle Diaboliques, suggerendo l'unità di tono della raccolta, ma anche mostra l'ideale di una rappresentazione tragica (ossia vera) del male nascosto per svelare l'aspetto diabolico della modernità.

Mathilde BERTRAND («Satan...pour rire»: les "Diaboliques" au miroir de Baudelaire, pp. 175-190) ricorda che quando anche Barbey è stato accusato nel 1874 di oltraggio alla morale per le Diaboliques, usa per difendersi gli stessi argomenti che aveva utilizzato a favore di Baudelaire, parlando di una moralizzazione attraverso il terrore, ed ottiene del resto lo stesso insuccesso. Anche l'articolo sui Paradis artificiels, espressamente richiesto da Baudelaire che sperava in una arringa difensiva, ha dei punti di contatto con la prefazione delle Diaboliques. Così come Barbey vede in Baudelaire uno scrittore ironico, così scrive per la propria opera una préface disinvolta che l'A. ritiene in contrasto col tono delle novelle. 
Christophe CHAGUINIAN (La modernité comme barrière à la compréhension des «Diaboliques», pp. 191-200) mostra le ragioni di un'incomprensione sull'etica delle Diaboliques, che ha suscitato il processo per oltraggio alla morale. L'A. spiega come Barbey sia legato a una visione del cattolicesimo invalsa fino alla prima metà dell' 800 e retta sul concetto di Male e della tentazione e sul terrore della punizione divina; mentre a partire dalla seconda metà l'immagine di Dio è prevalentemente legata a una visione di amore e di perdono. Nel secondo ' 800 , quindi, come per i moderni, è più difficile la comprensione di un'opera come le Diaboliques, in cui non si ritrova più niente di diabolico; mentre Barbey aveva in mente la trasgressione nei confronti dello spirito, ossia del comandamento di Dio.

10 Myriam watTHEE-DeLmotTe (Construire le contemporain par la fiction: la performativité littéraire chez Barbey d'Aurevilly, pp. 201-210) si sofferma sulla forma narrativa di Barbey, fondata su di un tempo discontinuo e a più voci, con una funzione performativa fondata sul modo in due tempi del rituale di lutto: commemorazione e separazione dal mondo antico, per impedire che il passato sia dimenticato e attraverso questo produrre un'azione innovatrice e trasformatrice.

11 Caroline SIDI (Barbey d'Aurevilly et La Fontaine: le tropisme moderne d'un classique, pp. 211-228) nota come La Fontaine sia incorporato in Barbey, che lo dichiara peraltro apertamente, arricchendo il bestiario di nuove specie. L'A. mostra come entrambi giochino con eguale libertà con le loro fonti, e come Barbey apprezzi l'indipendenza fiera e moderna dell'altro, pur se in La Fontaine la natura è prescrittiva, mentre Barbey mette in scena personaggi irriducibili alle classificazioni.

12 Michel BRIX, (Barbey, Flaubert et l'avenir du roman, pp. 229-242) ricorda gli articoli che Barbey ha dedicato all'opera di Flaubert, e nel suo giudizio generalmente negativo trova una conformità col giudizio della maggior parte dei contemporanei. In ogni caso, mentre Barbey apprezza dell'opera Mme Bovary almeno il personaggio di Emma, si convince di un Flaubert descrittore e privo di capacità narrativa con Salammbô. L'A. in conclusione fa notare come proprio quei difetti che individuava Barbey pongono Flaubert alle origini della finzione contemporanea.

Marie-Christine NATTA (Le vieil or et l'habit noir: Barbey d'Aurevilly et Baudelaire, pp. 243-256) prende le mosse dal primo incontro tra i due autori, nella redazione di «Le Pays» del 1853, per mostrare, nella diversità del loro abbigliamento, sobriamente nero quello di Baudelaire, vistoso e colorato quello di Barbey, un comune atteggiamento di dandy. Mentre però con l'adozione del nero, pur arricchito da dettagli di eleganza e singolarità, Baudelaire significa l'accettazione della sua epoca, Barbey segnala la sua nostalgia del passato. Ciò nonostante, è proprio il dandismo alle origini di una mutua comprensione e di un'amicizia che si incrinerà solo al momento della stroncatura di Poe da un punto di vista morale da parte di Barbey. Grazie ai loro abiti, entrambi si riconoscono eroici «stoïciens de boudoir».

14 Francesco SPANDRI (Les ridicules du temps démocratique, pp. 257-270) ripercorre la storia del testo del titolo, uscito a puntate su «Le Nain jaune» e caratterizzato, com'è naturale del resto in una simile pubblicazione, da un tono fortemente satirico. La critica è ormai quella di costume, contro un secondo Impero che scivola verso la società liberale e la democrazia, vista come una manifestazione dell'Errore in senso morale. La società appare malata, e proprio per questo è ridicola: ridicolo morale, naturalmente, la cui percezione rappresenta l'atto di resistenza. L'A. mostra in questa percezione il concetto 
di modernità di Barbey, che lo estende anche al «ridicolo letterario», di testi ormai fondati sulla fatuità e la vanità.

Reto zÖLLNER (Barbey d'Aurevilly et «le livre ouvert» de l'écriture et de la main, pp. 271-284) vede la modernità di Barbey come odio di ciò che è moderno, e riconosce la sua visione del mondo in un universo di segni da decifrare, dominati dal chiaroscuro tra scienza e mistero. Un'analisi relativa alle pagine sul testo di chiromanzia del capitano d'Arpentigny in Les CEuvres et les Hommes, e al passo di Une vieille maitresse sulla mano di Hermengarde, permette all'A. di delineare una corrispondenza tra la chiromanzia e la scrittura, che, anche nei suoi aspetti di grafia, è percepita da Barbey come mezzo di interpretazione di un'anima.

Herman HOFER (Quand Salomé réécrit Barbey, pp. 285-296) contro l'opinione diffusa che Barbey non abbia avuto una vera posterità letteraria, ne ricorda l'influenza su Proust, Bernanos, Bourges, Bloy e Lorrain tra i maggiori, per indicare poi una lunga serie di scrittori del Novecento europeo, tra cui Artaud, Musil, Beauvoir. Ma l'A. concede lo spazio maggiore al rapporto tra Louise Read e Wedekind, e al passaggio attraverso di lei di elementi aurevilliani nel drammaturgo tedesco.

Gaël PRIGENT (Barbey: un anti-moderne vu par les modernes, pp. 297-312) osserva come Barbey sia poco o nulla citato da autori cattolici o di formazione cattolica da cui ci si attenderebbe il contrario, come Hello, Péguy, Claudel, Bernanos, Jammes, Gide o Mauriac. Green cita appena Les Diaboliques in relazione a Bernanos. È invece in Proust che Barbey è visto come un modello artistico, citato a più riprese, e ammirazione manifestano Julien Gracq e Paul Morand. L'A. si sofferma quindi sull'interesse manifestato in tempi più recenti da altri autori tra cui Maurice Dantec e François Taillandier.

Pascale AURAIX-JONCHIÈRE (Le Bonheur dans le crime: «une bulle de savon qui grandit toujours et qui ne crève jamais!», pp. 313-324) compie un'analisi del romanzo di Jacqueline Harpman, Le Bonheur dans le crime, che si apre su di un'epigrafe dell'omonima novella di Barbey, mettendo in luce la comunanza formale nella struttura dei due testi, anche a livello di reti metaforiche.

Philippe BERTHIER (Barbey à travers les âges: pour une lecture aurevillienne de Philippe Muray, pp.325-334) conclude il volume con un commosso omaggio a Philippe Muray, scomparso nel maggio 2006, in cui l'A. vede il Barbey della nostra modernità, il fustigatore del pensiero unico. 